PREPARED FOR THE U.S. DEPARTMENT OF ENERGY, UNDER CONTRACT DE-AC02-76CH03073

PPPL-3945

PPPL-3945

UC-70

Approximate Integrals of rf-driven Particle Motion in Magnetic Field

by

I.Y. Dodin and N.J. Fisch

April 2004

$\left.\stackrel{M}{M}\right|_{\substack{\text { PRInCETON PLASIMA } \\ \text { PHYSICS LABORATORY }}} ^{D}$

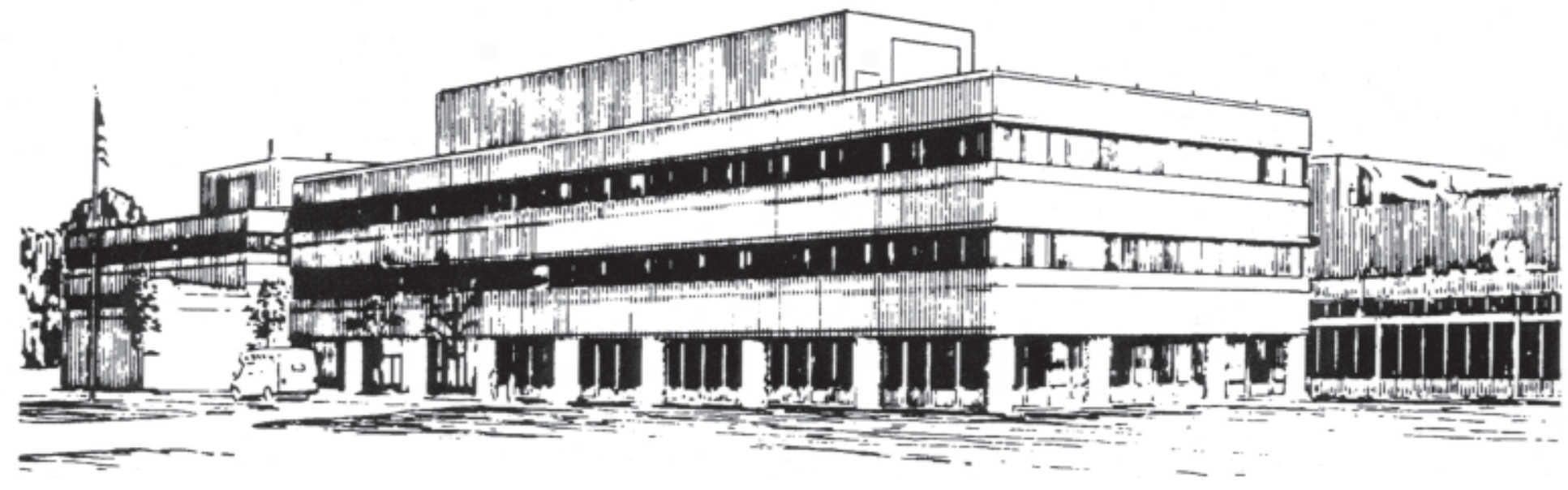

PRINCETON PLASMA PHYSICS LABORATORY PRINCETON UNIVERSITY, PRINCETON, NEW JERSEY 


\section{PPPL Reports Disclaimer}

This report was prepared as an account of work sponsored by an agency of the United States Government. Neither the United States Government nor any agency thereof, nor any of their employees, makes any warranty, express or implied, or assumes any legal liability or responsibility for the accuracy, completeness, or usefulness of any information, apparatus, product, or process disclosed, or represents that its use would not infringe privately owned rights. Reference herein to any specific commercial product, process, or service by trade name, trademark, manufacturer, or otherwise, does not necessarily constitute or imply its endorsement, recommendation, or favoring by the United States Government or any agency thereof. The views and opinions of authors expressed herein do not necessarily state or reflect those of the United States Government or any agency thereof.

\section{Availability}

This report is posted on the U.S. Department of Energy's Princeton Plasma Physics Laboratory Publications and Reports web site in Fiscal Year 2004. The home page for PPPL Reports and Publications is: http://www.pppl.gov/pub_report/

DOE and DOE Contractors can obtain copies of this report from:

U.S. Department of Energy

Office of Scientific and Technical Information

DOE Technical Information Services (DTIS)

P.O. Box 62

Oak Ridge, TN 37831

Telephone: (865) 576-8401

Fax: (865) 576-5728

Email: reports@adonis.osti.gov

This report is available to the general public from:

National Technical Information Service

U.S. Department of Commerce

5285 Port Royal Road

Springfield, VA 22161

Telephone: $1-800-553-6847$ or

(703) $605-6000$

Fax: (703) 321-8547

Internet: http://www.ntis.gov/ordering.htm 


\title{
Approximate integrals of rf-driven particle motion in magnetic field
}

\author{
I.Y. Dodin and N.J. Fisch \\ Princeton Plasma Physics Laboratory, Princeton, NJ 08543, USA
}

\begin{abstract}
For a particle moving in nonuniform magnetic field under the action of an $\mathrm{rf}$ wave, ponderomotive effects result from rf-driven oscillations nonlinearly coupled with Larmor rotation. Using Lagrangian and Hamiltonian formalism, we show how, despite this coupling, two independent integrals of the particle motion are approximately conserved. Those are the magnetic moment of free Larmor rotation and the quasi-energy of the guiding center motion parallel to the magnetic field. Under the assumption of nonresonant interaction of the particle with the rf field, these integrals represent adiabatic invariants of the particle motion.
\end{abstract}

\section{INTRODUCTION}

Under the action of an intense rf drive, charged particles undergo fast oscillations superimposed on the average drift motion. If the drift is slow enough and the amplitude of particle oscillations is small compared with the characteristic spatial scale of the applied external fields, then the particle average motion can be described in the framework of the guiding center approach. In this case, the average effect of the rf drive can approximately be replaced by the particle interaction with an effective potential [1,2].

One of the applications where the guiding center approximation finds use is the problem of the particle motion under the action of intense rf radiation superimposed on the particle interaction with a static nonuniform magnetic field [2]. An interesting variation of this effect with important consequences occurs when the static field is nonuniform [3]. In this case, the particle motion consists not only of the rf-driven oscillations, but also of the Larmor rotation in the plane perpendicular to magnetic field lines, accompanied by the diamagnetic acceleration of the particle guiding center parallel to the magnetic field. These types of motion can be easily studied separately when either the rf field or the magnetic field is negligible: In the absence of rapidly oscillating rf fields, it can be shown that the magnetic moment associated with particle Larmor rotation, $\mu=m v_{\perp}^{2} / 2 B_{0}$, represents an adiabatic invariant $[4,5]$, where $\frac{1}{2} m v_{\perp}^{2}$ is the energy of particle motion transverse to the magnetic field $\mathbf{B}_{0}$. In the other case, when only the rf field is present, the adiabatic invariance of the quantity $\frac{1}{2} m\langle v\rangle^{2}+\Phi$ can be proven (see, e.g., [6]), where $\langle v\rangle$ is the guiding center velocity, and $\Phi$ is the so-called ponderomotive potential $[1,2]$ given by

$$
\Phi=\frac{e^{2}\left|\mathbf{E}_{\mathrm{rf}}^{(0)}\right|^{2}}{4 m \omega^{2}}
$$

where $e$ and $m$ are the electric charge and the mass of the particle, $\mathbf{E}_{\mathrm{rf}}^{(0)}$ and $\omega$ are the complex amplitude and the frequency of the rf field.

In the case when both magnetic and rf fields are present, the question about the adiabatic invariants conservation becomes nontrivial because of possible coupling between the Larmor rotation and the rf-driven motion. As the Larmor frequency becomes comparable to the frequency of the rf field, conventional hierarchy of adiabatic invariants 
[6] cannot be developed, and, in principle, chaotic motion may result from nonlinear interaction between the two types of oscillations.

Usually, when the motion of an rf-driven particle in a magnetic field is studied, the two approximate integrals are derived [2]. Those are the magnetic moment associated with the particle free Larmor rotation $\mu=m \mathbf{v}_{\mathrm{f}, \perp}^{2} / 2 B_{0}$ (here $\mathbf{v}_{\mathrm{f}, \perp}=\mathbf{v}_{\perp}-\mathbf{v}_{\mathrm{rf}, \perp}$ is the velocity additional to the velocity of the rf-driven oscillations $\mathbf{v}_{\mathrm{rf}}$ ) and the particle "quasi-energy"

$$
\mathcal{E}=\frac{m\left\langle v_{\|}\right\rangle^{2}}{2}+\mu B_{0}+\Phi,
$$

with the effective potential $\Phi$ given by

$$
\Phi=\sum_{\nu=0, \pm 1} \frac{e^{2}\left|E_{\nu}^{(0)}\right|^{2}}{4 m \omega(\omega+\nu \Omega)}
$$

Here $E_{\nu}^{(0)}$ is the amplitude of the electric rf field component with polarization $\boldsymbol{\tau}_{\nu}$,

$$
\boldsymbol{\tau}_{ \pm 1}=\left(\mathbf{x}^{0} \pm i \mathbf{y}^{0}\right) / \sqrt{2}, \quad \boldsymbol{\tau}_{0}=\mathbf{z}^{0}
$$

where $\mathbf{x}^{0}$ and $\mathbf{y}^{0}$ are the unit vectors in the plane perpendicular to the magnetic field $\mathbf{B}_{0} \approx \mathbf{z}^{0} B_{0}(z)$, smooth on the scale of the oscillations amplitude; $\Omega=e B_{0} / m c$ is the Larmor frequency.

Though $\mu$ is often claimed to be an adiabatic invariant $[2,7,8]$, this statement, rather than proven rigorously, is usually made by analogy with the case of free Larmor rotation at zero rf field (see though the discussion in [9]). Consequently, conservation of $\mu$ is never examined analytically (for numerical and experimental studies, see [7, 8]). Moreover, it remains unclear exactly what is the nature of the integral (2) and what are the approximations under which $\mathcal{E}$ can be considered as a conserved quantity.

These shortcomings of the conventional consideration result from the intrinsic limitations of the approach used for deriving the average ponderomotive force. Namely, the guiding center motion equations are often obtained by direct averaging of the true motion equations, Taylor-expanded with respect to the spatial coordinate (see, e.g., [2, 10-13]). The potential form of the ponderomotive force in this case is not deduced directly — it is rather guessed (while the proof follows), which makes the complicated averaging procedure even more unclear. What we show, however, is that there exists an alternative, physically intuitive, formally simple, and clear in derivation Lagrangian approach, leading to the same expression for the average force "seen" by a slowly drifting particle.

The purpose of the present paper is, first, to present a simple Lagrangian derivation of the known conservation laws for $\mu$ and $\mathcal{E}$; second, to give a systematic Lagrangian and Hamiltonian formulation of the particle average motion; and, third, to demonstrate how such a treatment gives the conditions under which $\mu$ and $\mathcal{E}$ can approximately be considered as adiabatic invariants. The paper is organized as follows: In Sec. II we show how the average potential (3) can be derived naturally and the conservation of $\mu$ and $\mathcal{E}$ can be proved in the framework of the Lagrangian approach. A more detailed calculation involving the Hamiltonian analysis is given in Sec III, which demonstrates the connection between the approximate integrals of the rf-driven particle motion and the theory of adiabatic invariants. We show that $\mu$ and $\mathcal{E}$ represent adiabatic invariants of the particle motion only under the assumption of negligible heating of 
a particle at high-order resonances, which always takes place when the particle travels along a nonuniform magnetic field under the action of an rf drive. In Sec IV, we summarize our main ideas. Some supplementary calculations are given in Appendices A and B.

\section{GUIDING CENTER LAGRANGIAN}

To study the average motion of a charged particle under the action of rf radiation in a dc magnetic field, let us first concretely define the guiding center approximation. The key condition under which particle dynamics can be readily averaged over fast oscillations is that the particle displacement on a oscillation time scale is small compared with the scale of the external field. Hence, the obvious conditions which are required can be put as

$$
\frac{r_{\sim}}{\Delta} \ll 1, \quad \frac{|\langle\mathbf{v}\rangle|}{\omega \Delta} \ll 1, \quad \frac{|\langle\mathbf{v}\rangle|}{\Omega \Delta} \ll 1,
$$

where $r_{\sim}$ is the amplitude of particle oscillations and $\Delta$ is the least characteristic spatial scale of electromagnetic field. In addition to those, however, one also needs the drift motion to remain slow in comparison with the beating period between Larmor rotation and rf-driven oscillations $\tau_{\mathrm{b}}=2 \pi /|\omega-\Omega|$, and that $\tau_{\mathrm{b}}$ itself varies smoothly along the particle trajectory:

$$
\frac{\left\langle v_{z}\right\rangle}{|\omega-\Omega| \Delta} \ll 1, \quad \frac{\left\langle v_{z}\right\rangle}{(\omega-\Omega)^{2}} \frac{d(\omega-\Omega)}{d z} \ll 1
$$

To develop the guiding center description under the conditions (5), (6), first consider the expression for the action

$$
S=\int_{t_{1}}^{t_{2}} L d t
$$

where $L$ is the Lagrangian of the particle motion. Consider the time scale $\Delta t=t_{2}-t_{1}$ large compared to $\tau_{\mathrm{b}}$. Then, the major contribution to the action $S$ (linear on $\Delta t$ ) is provided by the time-averaged part of the Lagrangian, $\langle L\rangle$, while the contribution of the oscillatory Lagrangian into the integral (7) remains small. Thus, the action $S$ is approximately given by $S=\int_{t_{1}}^{t_{2}}\langle L\rangle d t$, from where it follows that $L_{\mathrm{d}} \equiv\langle L\rangle$ can be treated as the Lagrangian of the guiding center motion.

To calculate $L_{\mathrm{d}}$, consider the full Lagrangian of a particle moving in a static magnetic field $\mathbf{B}_{0}=\nabla \times \mathbf{A}_{0}$ under the action of $\mathrm{rf}$ drive governed by the vector potential $\mathbf{A}_{\mathrm{rf}}$ :

$$
L=\frac{m v^{2}}{2}+\frac{e}{c}\left(\mathbf{v} \cdot\left(\mathbf{A}_{0}+\mathbf{A}_{\mathrm{rf}}\right)\right)
$$

Take $\epsilon$ to be the largest among the small parameters defined in Eqs. (5), (6). In the limit $\epsilon \ll 1$, the vector potential $\mathbf{A}_{0}(\mathbf{r})$ can be approximated with a linear function of the particle transverse displacement:

$$
\mathbf{A}_{0}(\mathbf{r})=B_{0}(z)\left(\mathbf{z}^{0} \times \mathbf{r}\right) / 2+\mathcal{O}(\epsilon)
$$

(see also [14]). Let us denote the rf-driven oscillatory displacement with $\mathbf{r}_{\mathrm{rf}}$, and introduce the new coordinate $\mathbf{R}=\mathbf{r}-\mathbf{r}_{\mathrm{rf}}$ together with the corresponding velocity $\mathbf{V}=d \mathbf{R} / d t$ and the quiver velocity $\mathbf{v}_{\mathrm{rf}}=d \mathbf{r}_{\mathrm{rf}} / d t$. Then the 
Lagrangian (8) takes the form

$$
\begin{aligned}
& L=\frac{m V^{2}}{2}+\frac{e}{c}\left(\mathbf{V} \cdot \mathbf{A}_{0}(\mathbf{R})\right)+L_{\mathrm{rf}}+L_{\sim}, \\
& L_{\mathrm{rf}}=\frac{m v_{\mathrm{rf}}^{2}}{2}+\frac{e}{c}\left(\mathbf{v}_{\mathrm{rf}} \cdot \mathbf{A}_{\sim}\right), \\
& L_{\sim}=m \mathbf{V} \cdot \mathbf{p}_{\sim}+\frac{e}{c}\left(\mathbf{v}_{\mathrm{rf}} \cdot \mathbf{A}_{0}(\mathbf{R})\right)+\mathcal{O}(\epsilon),
\end{aligned}
$$

where $\mathbf{p}_{\sim}=m \mathbf{v}_{\mathrm{rf}}+(e / c) \mathbf{A}_{\sim}=m \mathbf{v}_{\mathrm{rf}, \perp}+(e / c) \mathbf{A}_{\sim, \perp}+\mathcal{O}(\epsilon)$ is the the oscillatory momentum, and $\mathbf{A}_{\sim}=\mathbf{A}_{\mathrm{rf}}+\mathbf{A}_{0}\left(\mathbf{r}_{\mathrm{rf}}\right)$ is the the oscillatory vector potential "seen" by the particle.

In comparison with traditional averaging of motion equations (see, e.g., $[2,10-13])$, the advantage of the Lagrangian approach consists of the fact that, in the guiding center Lagrangian, it is enough to keep only the zeroth-order terms with respect to $\epsilon$. (The ponderomotive force, which is of the first order in $\epsilon$, readily appears in the motion equation, as the guiding center Lagrangian is differentiated with respect to $Z \equiv R_{z}$.) In the limit of zero $\epsilon$, the function $L_{\sim}$ represents a full time derivative, $L_{\sim}=d\left(\mathbf{R}_{\perp} \cdot \mathbf{p}_{\sim, \perp}\right) / d t+\mathcal{O}(\epsilon)$, and thus can be taken out from the original Lagrangian. Therefore, the particle motion can be equivalently described in terms of the alternative Lagrangian function

$$
\begin{aligned}
& \mathcal{L}=\frac{m \dot{Z}^{2}}{2}+L_{\perp}+L_{\mathrm{rf}}+\mathcal{O}(\epsilon), \\
& L_{\perp}=\frac{m V_{\perp}^{2}}{2}+\frac{e}{c}\left(\mathbf{V}_{\perp} \cdot \mathbf{A}_{0}\left(\mathbf{R}_{\perp}, Z\right)\right) .
\end{aligned}
$$

From the form of the Lagrangian (13), it can be concluded that, in the limit $\epsilon \rightarrow 0$, a particle drifts along a magnetic field line with velocity $\left\langle v_{z}\right\rangle=\dot{Z}$, undergoes Larmor rotation in variables $\left(\mathbf{R}_{\perp}, \mathbf{V}_{\perp}\right)$ and experiences a ponderomotive force $\nabla L_{\mathrm{rf}}$. To derive the Lagrangian of the longitudinal drift motion, let us average the expression (13) over both Larmor and rf-driven oscillations, as well as over the beating between the two. Since $L_{\perp}$ has a form of the Lagrangian of Larmor motion in variables $\left(\mathbf{R}_{\perp}, \mathbf{V}_{\perp}\right)$, it can be shown (Appendix A) that, after omitting the full time derivative,

$$
\left\langle L_{\perp}\right\rangle=-\mu B_{0}, \quad \mu=\text { const. }
$$

Let us derive now the expression for the time-averaged function $L_{\mathrm{rf}}$. Under the action of an rf field a particle undergoes oscillations, which can be represented in the complex form as $\mathbf{r}_{\mathrm{rf}}=\left(-e / m \omega^{2}\right) \mathbf{T} \mathbf{E}_{\mathrm{rf}}$, where the tensor $\mathbf{T}$, for $\mathbf{E}_{\mathrm{rf}} \propto \exp (-i \omega t)$, is given by

$$
\mathbf{T}=\left(\begin{array}{ccc}
\frac{1}{1-b^{2}} & \frac{i b}{1-b^{2}} & 0 \\
\frac{-i b}{1-b^{2}} & \frac{1}{1-b^{2}} & 0 \\
0 & 0 & 1
\end{array}\right), \quad b=\Omega / \omega
$$

Consider the most general expression for the rf field:

$$
\mathbf{E}_{\mathrm{rf}}=\operatorname{Re}\left(E_{+}^{(0)} \boldsymbol{\tau}_{+}+E_{-}^{(0)} \boldsymbol{\tau}_{-}+E_{\|}^{(0)} \boldsymbol{\tau}_{0}\right) e^{-i \omega t}
$$

where $E_{\nu}^{(0)}$ are some arbitrary complex amplitudes, $\boldsymbol{\tau}_{\nu}$ are the polarization vectors defined according to Eq. (4). In this case, one has

$$
\left\langle L_{\mathrm{rf}}\right\rangle=\frac{e^{2}}{4 m \omega^{2}}\left\{\left|\mathbf{T} \mathbf{E}_{\mathrm{rf}}\right|^{2}-2 \operatorname{Re}\left[\mathbf{E}_{\mathrm{rf}}^{*} \cdot \mathbf{T} \mathbf{E}_{\mathrm{rf}}\right]+b \mathbf{z}^{0} \cdot \operatorname{Im}\left[\left(\mathbf{T}^{*} \mathbf{E}_{\mathrm{rf}}^{*}\right) \times\left(\mathbf{T} \mathbf{E}_{\mathrm{rf}}\right)\right]\right\}
$$


Since

$$
\mathbf{T E}_{\mathrm{rf}}=\frac{\mathbf{x}^{0}}{\sqrt{2}}\left(\frac{E_{+}^{(0)}}{1+b}+\frac{E_{-}^{(0)}}{1-b}\right)+\frac{i \mathbf{y}^{0}}{\sqrt{2}}\left(\frac{E_{+}^{(0)}}{1+b}-\frac{E_{-}^{(0)}}{1-b}\right)+\mathbf{z}^{0} E_{\|}^{(0)}
$$

it can be shown that

$$
\begin{aligned}
& \left|\mathbf{T} \mathbf{E}_{\mathrm{rf}}\right|^{2}=\frac{\left|E_{+}^{(0)}\right|^{2}}{(1+b)^{2}}+\frac{\left|E_{-}^{(0)}\right|^{2}}{(1-b)^{2}}+\left|E_{\|}^{(0)}\right|^{2}, \\
& \operatorname{Re}\left[\mathbf{E}_{\mathrm{rf}}^{*} \cdot \mathbf{T} \mathbf{E}_{\mathrm{rf}}\right]=\frac{\left|E_{+}^{(0)}\right|^{2}}{1+b}+\frac{\left|E_{-}^{(0)}\right|^{2}}{1-b}+\left|E_{\|}^{(0)}\right|^{2}, \\
& \operatorname{Im}\left[\left(\mathbf{T}^{*} \mathbf{E}_{\mathrm{rf}}^{*}\right) \times\left(\mathbf{T} \mathbf{E}_{\mathrm{rf}}\right)\right]_{z}=\frac{\left|E_{+}^{(0)}\right|^{2}}{(1+b)^{2}}-\frac{\left|E_{-}^{(0)}\right|^{2}}{(1-b)^{2}} .
\end{aligned}
$$

Substituting those into Eq. (18), one gets

$$
\left\langle L_{\mathrm{rf}}\right\rangle=-\frac{e^{2}}{4 m \omega^{2}}\left\{\frac{\left|E_{+}^{(0)}\right|^{2}}{1+b}+\frac{\left|E_{-}^{(0)}\right|^{2}}{1-b}+\left|E_{\|}^{(0)}\right|^{2}\right\}=-\Phi,
$$

where $\Phi$ is the ponderomotive potential defined according to Eq. (3).

Hence, finally, the expression for the guiding center Lagrangian can be put in the form

$$
L_{\mathrm{d}}=\frac{m \dot{Z}^{2}}{2}-\mu B_{0}(Z)-\Phi(Z)+\langle\mathcal{O}(\epsilon)\rangle
$$

yielding a motion equation in a potential form

$$
m \frac{d^{2} Z}{d t^{2}} \approx-\frac{d}{d Z}\left(\mu B_{0}(Z)+\Phi(Z)\right)
$$

which conserves the quasi-energy (2). Indeed, $\mathcal{E}$ coincides with the drift Hamiltonian of a particle, $H_{\mathrm{d}}=m \dot{Z}^{2}-L_{\mathrm{d}}$, $\dot{Z}=\left\langle v_{\|}\right\rangle$, and, since $\partial H_{\mathrm{d}} / \partial t=0$, the value of $\mathcal{E}$ represents an integral of the guiding center motion..

\section{ACTION-ANGLE VARIABLES}

As shown above, the two approximate integrals of the guiding center motion, $\mu$ and $\mathcal{E}$, exist for a particle undergoing Larmor rotation under the action of an rf field. In this section, we show how these integrals appear naturally from the Hamiltonian description of the ponderomotive effects (see also [9], where the rf field is treated as a perturbation). To be more precise, what is shown below is that, under certain conditions, $\mu$ and $\mathcal{E}$ can be considered as adiabatic invariants of the particle motion.

To proceed, let us develop the Hamiltonian formalism for particle dynamics starting from the Lagrangian (13). The canonical momentum of the motion, additional to the rf-driven oscillations, is given by $\mathbf{P}=m \mathbf{V}+(e / c) \mathbf{A}_{0}(\mathbf{R})$, and the Hamiltonian function can be put in the intuitively expected form

$$
H=\frac{P_{z}^{2}}{2 m}+\frac{1}{2 m}\left(\mathbf{P}_{\perp}-\frac{e}{c} \mathbf{A}_{0}\left(\mathbf{R}_{\perp}, Z\right)\right)^{2}-L_{\mathrm{rf}}(Z, t)+\mathcal{O}(\epsilon),
$$

where Larmor rotation in the variables $\left(\mathbf{R}_{\perp}, \mathbf{P}_{\perp},\right)$ is separated (at least, locally) from the rf-driven oscillations and the average motion parallel to magnetic field. After the canonical transformation to the Larmor guiding center variables (see, e.g., $[6,15]$ ), the equivalent Hamiltonian takes the form

$$
\bar{H}=\frac{P_{z}^{2}}{2 m}+\Omega(Z) P_{\phi}-L_{\mathrm{rf}}(Z, t)+\epsilon \Delta \bar{H}
$$


where $P_{\phi}=(m c / e) \mu$ is the action variable corresponding to the canonical angle $\phi$ standing for the Larmor phase of the particle, $\Delta \bar{H}$ is a periodic function of $\phi$ and $t$ (since the original particle Lagrangian is periodic with respect to these variables).

Consider now the the action (7) written as

$$
S=\int P_{z} d Z+P_{\phi} d \phi-\bar{H} d t
$$

From this representation [16], it follows that one can treat the quantity $-\bar{H}$ as a canonical momentum with the time $t$ as the corresponding canonical coordinate, while the pair $\left(-P_{z}, Z\right)$ is treated as the new Hamiltonian $\widehat{H}$ and the new "time": $\widehat{H}=-P_{z}\left(\phi, P_{\phi} ; t,-\bar{H} ; Z\right)$. Assuming, for clarity, that $P_{z}$ is positive, one gets

$$
\widehat{H}=-\sqrt{2 m\left(\bar{H}+L_{\mathrm{rf}}(Z, t)-\Omega P_{\phi}\right)}+\epsilon \Delta \widehat{H},
$$

with small term $\epsilon \Delta \widehat{H}$, periodic in $t$ and $\phi$.

Let us perform another canonical transformation to represent the Hamiltonian function in terms of the action variable

$$
P_{\varphi}=\frac{1}{2 \pi} \oint \bar{H} d t
$$

and the corresponding angle variable $\varphi$, yet to be defined. To do so, consider the generating function

$$
F\left(P_{\varphi}, t\right)=-\int_{0}^{t} \bar{H} d t=-P_{\varphi} \omega t+F_{\sim}
$$

with $F_{\sim}$ having zero time average. The new Hamiltonian $\mathcal{H}=\widehat{H}+\partial F / \partial Z$ is given by $\mathcal{H}=\mathcal{H}_{0}+\epsilon \mathcal{H}_{\sim}$, where

$$
\mathcal{H}_{0}=-\sqrt{2 m\left(\omega P_{\varphi}-\Phi(Z)-\Omega P_{\phi}\right)}
$$

and $\mathcal{H}_{\sim}$ is periodic with respect to $\phi$ and $t$. Since

$$
\varphi=\frac{\partial F}{\partial P_{\varphi}}=-\omega t-\mathcal{O}(\epsilon)
$$

where the second term is periodic in $t, \mathcal{H}_{\sim}$ also appears to be periodic in $\varphi$. Finally, introducing the vector action $\mathbf{J}=\left(P_{\phi}, P_{\varphi}\right)$ and the corresponding angle variable $\boldsymbol{\theta}=(\phi, \varphi)$, one can put the Hamiltonian $\mathcal{H}$ in the form

$$
\mathcal{H}=\mathcal{H}_{0}(\mathbf{J} ; Z)+\epsilon \mathcal{H}_{\sim}(\mathbf{J}, \boldsymbol{\theta} ; Z)
$$

where the small term $\mathcal{H}_{\sim}$ is periodic in $\boldsymbol{\theta}$ and thus can be represented as Fourier series

$$
\mathcal{H}_{\sim}=\sum_{\mathbf{n}} \mathcal{H}_{\mathbf{n}}(\mathbf{J} ; Z) \exp (i \mathbf{n} \cdot \boldsymbol{\theta})
$$

with summation taken over all possible pairs of integers $\mathbf{n}=\left(n_{\phi}, n_{\varphi}\right)$.

Consider now another canonical transformation to the new variables $(\overline{\boldsymbol{\theta}}, \overline{\mathbf{J}})$ with the generating function given by

$$
\mathcal{S}(\overline{\mathbf{J}}, \boldsymbol{\theta})=\overline{\mathbf{J}} \cdot \boldsymbol{\theta}+\sum_{\mathbf{n}, k} \epsilon^{k} \mathcal{S}_{\mathbf{n}, k}(\overline{\mathbf{J}} ; Z) \exp (i \mathbf{n} \cdot \boldsymbol{\theta})
$$


For small $\epsilon$, one can find such coefficients $\mathcal{S}_{\mathbf{n}}$, for which the new Hamiltonian $\overline{\mathcal{H}}$ represents a function of $\overline{\mathbf{J}}$ alone $($ see, e.g., [6] and the references therein). To the first order in $\epsilon$ one has

$$
\overline{\mathbf{J}}=\mathbf{J}+\epsilon \sum_{\mathbf{n} \neq 0} \mathbf{n}\left\langle v_{\|}\right\rangle \frac{\mathcal{H}_{\mathbf{n}}(\overline{\mathbf{J}} ; Z)}{\mathbf{n} \cdot \boldsymbol{\Xi}} \exp (i \mathbf{n} \cdot \overline{\boldsymbol{\theta}})
$$

(see also [9]). Here $\overline{\boldsymbol{\theta}}$ is considered a function of $\boldsymbol{\theta}$, and $\boldsymbol{\Xi}=\left\langle v_{\|}\right\rangle \partial \mathcal{H}_{0} / \partial \mathbf{J}=(\Omega,-\omega)$ is the frequency vector.

By construction, the new Hamiltonian $\overline{\mathcal{H}}(\overline{\mathbf{J}}, \overline{\boldsymbol{\theta}})$ does not depend on $\overline{\boldsymbol{\theta}}$ to any order in $\epsilon$. Thus, from the canonical equation $d \overline{\mathbf{J}} / d Z=-\partial \overline{\mathcal{H}} / \partial \overline{\boldsymbol{\theta}}$, it follows that $\overline{\mathbf{J}}$ is conserved with exponential precision if the above procedure can be realized (see below). In this case, the value of $\overline{\mathbf{J}}$ represents a so-called adiabatic invariant of the particle motion. As one can see from the definition of the action variable $\overline{\mathbf{J}}=\left(\bar{P}_{\phi}, \bar{P}_{\varphi}\right)$, the previously introduced quantities $\mu$ and $\mathcal{E}$ can be represented in the form

$$
\mu=(e / m c) \bar{P}_{\phi}+\mathcal{O}(\epsilon), \quad \mathcal{E}=\bar{P}_{\varphi} \omega+\mathcal{O}(\epsilon)
$$

and thus also represent approximate integrals of the particle motion. If evaluated away from the region of nonzero Hamiltonian of interaction $\epsilon \mathcal{H}_{\sim}$, the $\mathcal{O}(\epsilon)$ terms vanish. Therefore, after the particle has experienced a complete transition between the two regions of nonzero $\epsilon$, the overall changes of $\mu$ and $\mathcal{E}$ are exactly proportional to the changes of $\bar{P}_{\phi}$ and $\bar{P}_{\varphi}$ correspondingly:

$$
\Delta \mu=(e / m c) \Delta \bar{P}_{\phi} \approx 0, \quad \Delta \mathcal{E}=\omega \Delta \bar{P}_{\varphi} \approx 0
$$

Note, however, that under the condition of resonant interaction between the particle cyclotron motion and the rf field $\left(n_{\phi} \Omega=n_{\varphi} \omega\right)$ the canonic transformation (36) cannot be accomplished because of its singularity. Hence, the conservation laws (39) are violated, and a particle becomes capable of exchanging energy with the field. Such nonadiabatic interaction always takes place when $\Omega$ varies along the particle trajectory, so that the particle consecutively passes resonant regions corresponding to different $\mathbf{n}$. Let us estimate the change of action $\mathbf{J}$ as a particle crosses a resonance region $n_{\phi} \Omega=n_{\varphi} \omega$. Suppose $n_{\phi} / n_{\varphi} \neq \pm 1$, so that one may take $\mathcal{H}_{\mathbf{n}} \approx$ const. In this case, applying the steepest descent method when integrating the canonical equation for $\mathbf{J}$ and taking $B_{0} / B_{0}^{\prime}=L_{B} \approx$ const, one gets for $\mathbf{n} \neq 0$ :

$$
(\Delta \mathbf{J})_{\mathbf{n}} \approx 2 \epsilon \mathbf{n}\left|\mathcal{H}_{\mathbf{n}}\right| \sqrt{\pi \frac{\left|\left\langle v_{\|}\right\rangle\right| L_{B}}{n_{\varphi} \omega}} \cos \psi_{\mathbf{n}},
$$

where $\psi_{\mathbf{n}}$ is a constant determined by initial conditions. Note that, since $\epsilon \propto L_{B}^{-1}$, the change of the action variable scales like $L_{B}^{-1 / 2}$. If $L_{B}$ is large enough, so that $(\Delta \mathbf{J})_{\mathbf{n}} \ll \mathbf{J}$, then scattering on multiple resonances, crossed at random moments of time can be considered a diffusive process in the $\mathbf{J}$ space. Indeed, in average over $\psi_{\mathbf{n}}$ change of action, grows linearly with trace $Z$ :

$$
\left\langle(\Delta \mathbf{J})_{\Sigma}^{2}\right\rangle \sim Z \frac{|\Delta \mathbf{J}|^{2}}{\Delta z}
$$

where $|\Delta \mathbf{J}|$ is the characteristic (over $\mathbf{n}$ ) change of action (40), $\Delta z$ is the characteristic distance between the resonances. To ensure that $\left\langle(\Delta \mathbf{J})_{\Sigma}^{2}\right\rangle$ is small, it is necessary that the amplitues of harmonics $\left|\mathcal{H}_{\mathbf{n} \neq 0}\right|$ are sufficiently small. Only in this case, approximate conservation of adiabatic invariants (39) can be claimed. 
In the end, it is important to emphasize that, though the above consideration was developed for strictly periodic rf fields, it can also be extended to include more general situation of interest, that is the rf field consisting of non-commensurate multiple harmonics. In this case, the rf-driven motion no longer remains periodic, and the $P_{\varphi}$ conservation theorem must be revised. (Note that the loss of periodicity for the rf-driven motion does not impact the $\mu$ conservation theorem, since Larmor rotation stays periodic with the frequency $\Omega$.) For this situation, one must redefine the action $P_{\varphi}$ as the "energy" $\bar{H}$ averaged over some arbitrary time interval $\Delta \tau$, large compared to the correlation time of the rf-driven oscillatory motion $\tau_{\sim}$ :

$$
P_{\varphi}=\lim _{\Delta \tau / \tau_{\sim} \rightarrow \infty} \frac{1}{\Delta \tau} \int_{\Delta \tau} \bar{H} d t=\langle\mathcal{E}\rangle .
$$

As shown in Appendix B, for aperiodic processes, the quantity (42) represents an approximate integral of the particle motion (under the same stipulations as discussed above). Hence, the quasi-energy of a particle $\mathcal{E}$ and the (modified) magnetic moment $\mu$ are approximately conserved throughout particle motion in arbitrary nonresonant rf field under the condition of small $\epsilon$.

\section{SUMMARY}

In this paper, we showed how the Lagrangian formulation of ponderomotive effects can be used to derive the average potential. This formulation also makes physically and mathematically clear the origin of the well-known approximate integrals of the particle motion. Those include the magnetic moment $\mu$ of free Larmor rotation (additional to the externally driven motion), and the quasi-energy $\mathcal{E}$ of the guiding center motion parallel to the magnetic field. By developing the Hamiltonian formulation, we showed that $\mu$ and $\mathcal{E}$ represent adiabatic invariants of the particle motion only under the assumption of negligible heating at high-order resonances, which otherwise results in diffusive variations of these quantities. With minor reservations, the conservation of $\mu$ and $\mathcal{E}$ is preserved for both periodic and aperiodic high-frequency fields though, in the latter case, the periodicity of the particle motion may be lost completely.

The work was supported by the US DOE, under contract DE-AC0276-CHO3073.

\section{APPENDIX A: EXPRESSION FOR $\left\langle L_{\perp}\right\rangle$}

The Larmor rotation, additional to the rf-driven motion can be described in terms of the Larmor radius $\rho=\left|\mathbf{R}_{\perp}\right|$, the gyrophase $\phi$, and the corresponding canonical momentum $P_{\phi}=m \rho^{2}\left(\dot{\phi}+\frac{1}{2} \Omega\right)$. Using these variables, one can rewrite Eq. (14) in the form

$$
L_{\perp}=-\phi \frac{d P_{\phi}}{d t}-\mu B_{0}+\frac{d}{d t}\left(\phi P_{\phi}\right)
$$

where $\mu=m \mathbf{v}_{\mathrm{f}, \perp}^{2} / 2 B_{0}$. Note that

$$
\frac{d P_{\phi}}{d t}=\frac{d \mathcal{L}}{d \phi}=\sum_{n} i n \mathcal{C}_{n}(Z, \dot{Z}) \exp (i n \phi)
$$


where we used the Fourier transformation of $\mathcal{L}$ with respect to the angle variable $\phi$. The $\phi$-dependence of $\mathcal{L}$ can originate only from the inhomogeneity of the magnetic (and rf) field, which vanishes in the zeroth-order approximation in $\epsilon$, and thus $\mathcal{C}_{n}=\mathcal{O}(\epsilon)$. Also note that $d \phi / d t=\Omega+\mathcal{O}(\epsilon)$, and therefore the time-averaged derivative of $P_{\phi}$ is small compared to $\epsilon$ :

$$
\left\langle d P_{\phi} / d t\right\rangle=o(\epsilon)
$$

From the obtained result, it follows that $P_{\phi}\left(\right.$ or $\left.\mu=(e / m c) P_{\phi}\right)$ represents an approximate integral of the particle motion. (More careful discussion is given in Sec. III; see also [2, 9].)

The contribution of the first term in Eq. (A1) into the integral (7) taken over a large time $\Delta t=\mathcal{O}\left(\epsilon^{-1}\right)$ scales like $\mathcal{O}(1)$. Since the contribution of the $\mu B_{0}$ term appears to be of the order of $\epsilon^{-1}$, the first term in Eq. (A1) can be neglected and, omitting the full-time derivative, one can approximate the average Lagrangian function (A1) as

$$
\left\langle L_{\perp}\right\rangle=-\mu B_{0}
$$

where $\mu=$ const (see also [14]).

\section{APPENDIX B: APPROXIMATE INTEGRAL OF APERIODIC MOTION WITH A SLOWLY VARYING PARAMETER}

Consider a dynamic system governed by the Hamiltonian function $\mathcal{H}(\mathcal{Q}, \mathcal{P}, \lambda(t))$ with a parameter $\lambda(t)$ slowly varying in time $t$. Assume that, for $d \lambda / d t=0$, the system undergoes aperiodic oscillatory motion with a characteristic correlation time $\tau_{\sim}$. Assume also that these oscillations are statistically uniform on time scales large compared to $\tau_{\sim}$. Consider the action $J$, that is the canonical momentum $\mathcal{P}$ averaged over the fixed trajectory $\Delta \mathcal{Q}=\int_{\Delta T} d \mathcal{Q}$, along which the system travels during some large time $\Delta T \gg \tau_{\sim}$ :

$$
J=\lim _{\frac{\Delta T(\Delta \mathcal{Q})}{\tau \sim} \rightarrow \infty} \frac{1}{\Delta \mathcal{Q}} \int_{\Delta \mathcal{Q}} \mathcal{P}_{0} d \mathcal{Q}_{0}
$$

where the subindex 0 denotes quantities evaluated on the unperturbed trajectory with $d \lambda / d t=0$. The above-imposed requirement of statistical uniformity of the oscillatory motion guarantees that the averaging procedure is well-defined, so that the limiting value of the integral (B1) exists.

Let us prove that the action $J$ represents an approximate integral of the system, i.e. remains constant if $\lambda(t)$ changes slowly compared to unperturbed oscillations, in the sense that

$$
\epsilon=\Delta T\left|\frac{1}{\lambda} \frac{d \lambda}{d t}\right| \ll 1 .
$$

(The treatment of periodic oscillations, similar to the one given below, can be found in [16].) To do so, consider the time derivative

$$
\frac{d J}{d t}=\frac{1}{\Delta \mathcal{Q}} \int_{\Delta \mathcal{Q}} \frac{\partial \mathcal{P}_{0}}{\partial t} d \mathcal{Q}_{0}
$$


where we omitted the limit sign for clarity and made use of the fact that the limits of integration do not depend on time. Since the integration is performed over the unperturbed trajectory, $\mathcal{P}_{0}$ must be considered a function of $\mathcal{Q}_{0}$, parameter $\lambda$ and energy $E: \mathcal{P}_{0}=\mathcal{P}_{0}\left(\mathcal{Q}_{0} ; \lambda, E\right)$. Then

$$
\frac{d J}{d t}=\frac{1}{\Delta \mathcal{Q}} \int_{\Delta \mathcal{Q}}\left(\frac{\partial \mathcal{P}_{0}}{\partial \lambda} \frac{d \lambda}{d t}+\frac{\partial \mathcal{P}_{0}}{\partial E} \frac{d E}{d t}\right) d \mathcal{Q}_{0},
$$

where the partial derivatives can be obtained by differentiating the definition of the energy $E=\mathcal{H}(\mathcal{Q}, \mathcal{P} ; \lambda)$ :

$$
d E=\frac{\partial \mathcal{H}}{\partial \mathcal{Q}} d \mathcal{Q}+\frac{\partial \mathcal{H}}{\partial \mathcal{P}} d \mathcal{P}+\frac{\partial \mathcal{H}}{\partial \lambda} d \lambda
$$

Representing the above expression as the complete differential of $\mathcal{P}_{0}\left(\mathcal{Q}_{0} ; \lambda, E\right)$, one gets

$$
\frac{\partial \mathcal{P}_{0}}{\partial \lambda}=-\frac{\partial_{\lambda} \mathcal{H}_{0}}{\partial_{\mathcal{P}_{0}} \mathcal{H}_{0}}, \quad \frac{\partial \mathcal{P}_{0}}{\partial E}=\frac{1}{\partial_{\mathcal{P}_{0}} \mathcal{H}_{0}}
$$

where $\mathcal{H}_{0} \equiv \mathcal{H}\left(\mathcal{Q}_{0}, \mathcal{P}_{0}, \lambda\right)$. Let us use the Hamiltonian equation $d \mathcal{Q}_{0} / d t=\partial \mathcal{H}_{0} / \partial \mathcal{P}_{0}$ to rewrite the above integral in the following form:

$$
\frac{d J}{d t}=\frac{1}{\Delta \mathcal{Q}} \int_{\Delta T}\left(-\frac{\partial \mathcal{H}_{0}}{\partial \lambda} \frac{d \lambda}{d t}+\frac{d E}{d t}\right) d t,
$$

equivalent to

$$
\frac{d J}{d t}=\frac{\Delta T}{\Delta \mathcal{Q}}\left(\left\langle\frac{d E}{d t}\right\rangle-\frac{d \lambda}{d t}\left\langle\frac{\partial \mathcal{H}_{0}}{\partial \lambda}\right\rangle_{\lambda}\right),
$$

where the subindex $\lambda$ stands for averaging at fixed value of the parameter $\lambda$, and, in the first-order approximation in $\epsilon, d \lambda / d t$ is assumed constant on time $\Delta T$. Since

$$
\frac{d E}{d t}=\frac{d \mathcal{H}}{d t}=\frac{\partial \mathcal{H}}{\partial t}=\frac{\partial \mathcal{H}}{\partial \lambda} \frac{d \lambda}{d t}
$$

and $\lambda(t)$ is a slow function, we again take $d \lambda / d t$ out of averaging and consider the rest a function of fixed $\lambda$ and energy. The latter allows replacing $\mathcal{H}$ with $\mathcal{H}_{0}$, so that one gets $\langle d E / d t\rangle=(d \lambda / d t)\left\langle\partial \mathcal{H}_{0} / \partial \lambda\right\rangle_{\lambda}$. Finally,

$$
d J / d t=o(\epsilon)
$$

from where it follows that the action $J$ represents an approximate integral of motion at small $\epsilon$.

Note that, in the above derivation, we implicitly assumed that, if $\lambda(t)$ is changing slowly, the true phase-space trajectory can be adequately approximated by the trajectory with fixed $\lambda$ on the whole time interval $\Delta T$. For the situation discussed in the main text, this requirement is fulfilled due to the linearity of the particle local response to the rf fields. However, in a strongly nonlinear system, phase space trajectories may be unstable with respect to small variations of parameters, rendering invalid the above analysis.

[1] A. V. Gaponov and M. A. Miller 1958 Sov. Phys. JETP 7, 168. 
[2] H. Motz and C. J. H. Watson 1967 Advances in Electronics 23, 153.

[3] N. J. Fisch, J. M. Rax, and I. Y. Dodin 2003 Phys. Rev. Lett. 91, 205004.

[4] C. S. Gardner 1959 Phys. Rev. 115, 791.

[5] J. D. Jackson 1975 Classical electrodynamics. New York: Wiley.

[6] A. J. Lichtenberg and M. A. Lieberman 1992 Regular and Chaotic Dynamics, 2nd ed. New York: Springler Verlag.

[7] C. J. H. Watson and L. G. Kuo-Petravic 1968 Phys. Rev. Lett. 20, 1231.

[8] H. P. Eubank 1969 Phys. Fluids 12, 234.

[9] C. Grebogi, A. N. Kaufman, and R. G. Littlejohn 1979 Phys. Rev. Lett. 43, 1668.

[10] A. Kildal 1999 Contrib. Plasma Phys. 39, 349.

[11] B. M. Lamb, G. Dimonte, and G. J. Morales 1984 Phys. Fluids 27, 1401.

[12] G. Dimonte, B. M. Lamb, and G. J. Morales 1983 Plas. Phys. 25, 713.

[13] C. Litwin 1994 Phys. Plasmas 1, 515.

[14] I. S. Danilkin 1995 Plasma Phys. Rep. 21, 777.

[15] R. White, L. Chen, and Z. Lin 2002 Phys. Plasmas 9, 1890.

[16] L. D. Landau and E. M. Lifshitz 1960 Mechanics. New York: Pergamon Press. 


\section{External Distribution}

Plasma Research Laboratory, Australian National University, Australia

Professor I.R. Jones, Flinders University, Australia

Professor João Canalle, Instituto de Fisica DEQ/IF - UERJ, Brazil

Mr. Gerson O. Ludwig, Instituto Nacional de Pesquisas, Brazil

Dr. P.H. Sakanaka, Instituto Fisica, Brazil

The Librarian, Culham Laboratory, England

Mrs. S.A. Hutchinson, JET Library, England

Professor M.N. Bussac, Ecole Polytechnique, France

Librarian, Max-Planck-Institut für Plasmaphysik, Germany

Jolan Moldvai, Reports Library, Hungarian Academy of Sciences, Central Research Institute for Physics, Hungary

Dr. P. Kaw, Institute for Plasma Research, India

Ms. P.J. Pathak, Librarian, Institute for Plasma Research, India

Ms. Clelia De Palo, Associazione EURATOM-ENEA, Italy

Dr. G. Grosso, Instituto di Fisica del Plasma, Italy

Librarian, Naka Fusion Research Establishment, JAERI, Japan

Library, Laboratory for Complex Energy Processes, Institute for Advanced Study, Kyoto University, Japan

Research Information Center, National Institute for Fusion Science, Japan

Dr. O. Mitarai, Kyushu Tokai University, Japan

Dr. Jiangang Li, Institute of Plasma Physics, Chinese Academy of Sciences, People's Republic of China

Professor Yuping Huo, School of Physical Science and Technology, People's Republic of China

Library, Academia Sinica, Institute of Plasma Physics, People's Republic of China

Librarian, Institute of Physics, Chinese Academy of Sciences, People's Republic of China

Dr. S. Mirnov, TRINITI, Troitsk, Russian Federation, Russia

Dr. V.S. Strelkov, Kurchatov Institute, Russian Federation, Russia

Professor Peter Lukac, Katedra Fyziky Plazmy MFF UK, Mlynska dolina F-2, Komenskeho Univerzita, SK-842 15 Bratislava, Slovakia

Dr. G.S. Lee, Korea Basic Science Institute, South Korea

Institute for Plasma Research, University of Maryland, USA

Librarian, Fusion Energy Division, Oak Ridge National Laboratory, USA

Librarian, Institute of Fusion Studies, University of Texas, USA

Librarian, Magnetic Fusion Program, Lawrence Livermore National Laboratory, USA

Library, General Atomics, USA

Plasma Physics Group, Fusion Energy Research Program, University of California at San Diego, USA

Plasma Physics Library, Columbia University, USA

Alkesh Punjabi, Center for Fusion Research and Training, Hampton University, USA

Dr. W.M. Stacey, Fusion Research Center, Georgia Institute of Technology, USA

Dr. John Willis, U.S. Department of Energy, Office of Fusion Energy Sciences, USA

Mr. Paul H. Wright, Indianapolis, Indiana, USA 
The Princeton Plasma Physics Laboratory is operated by Princeton University under contract with the U.S. Department of Energy.

\author{
Information Services \\ Princeton Plasma Physics Laboratory \\ P.O. Box 451 \\ Princeton, NJ 08543
}

Phone: 609-243-2750

Fax: 609-243-2751

e-mail: pppl_info@pppl.gov

Internet Address: http://www.pppl.gov 